\title{
A large common-source outbreak of norovirus gastroenteritis in a hotel in Singapore, 2012
}

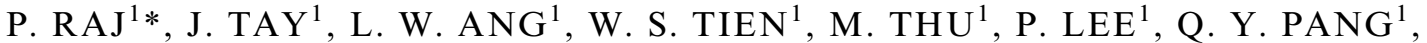 \\ Y. L. TANG ${ }^{1}$, K. Y. LEE ${ }^{1}$, S. MAURER-STROH ${ }^{2}$, V. GUNALAN ${ }^{2}$, J. CUTTER ${ }^{1}$ \\ AND K. T. GOH ${ }^{3}$ \\ ${ }^{1}$ Ministry of Health, Singapore, College of Medicine Building, Singapore \\ ${ }^{2} A * S T A R$ Singapore, Singapore \\ ${ }^{3}$ National University of Singapore, Singapore
}

Received 4 May 2016; Final revision 22 September 2016; Accepted 28 September 2016; first published online 28 October 2016

\section{SUMMARY}

An outbreak of gastroenteritis affected 453 attendees (attack rate $28 \cdot 5 \%$ ) of six separate events held at a hotel in Singapore. Active case detection, case-control studies, hygiene inspections and microbial analysis of food, environmental and stool samples were conducted to determine the aetiology of the outbreak and the modes of transmission. The only commonality was the food, crockery and cutlery provided and/or handled by the hotel's Chinese banquet kitchen. Stool specimens from 34 cases and 15 food handlers were positive for norovirus genogroup II. The putative index case was one of eight norovirus-positive food handlers who had worked while they were symptomatic. Several food samples and remnants tested positive for Escherichia coli or high faecal coliforms, aerobic plate counts and/or total coliforms, indicating poor food hygiene. This large common-source outbreak of norovirus gastroenteritis was caused by the consumption of contaminated food and/or contact with contaminated crockery or cutlery provided or handled by the hotel's Chinese banquet kitchen.

Key words: Common source, crockery, cutlery, epidemiological investigation, norovirus.

\section{INTRODUCTION}

Noroviruses are one of the leading causes of gastroenteritis in the world [1-4]. They can be transmitted by consumption of contaminated food and water or contact with contaminated fomites and environmental surfaces, and from person to person via the faecaloral route or through aerosolized virus particles generated during vomiting [5-7]. Norovirus is readily transmitted as the estimated median infectious dose

\footnotetext{
* Author for correspondence: Mr P. Raj, Ministry of Health, Singapore, College of Medicine Building, 16 College Road, Singapore 169854.

(Email: pream_raj@moh.gov.sg)
}

is only 18 viral particles [8]. Shedding can precede onset of symptoms, and persist for weeks after symptoms have resolved $[9,10]$. Asymptomatic individuals can shed similar viral loads as symptomatic individuals although they are less likely to transmit the virus. The difference in transmission is attributed to enhanced environmental seeding of the virus associated with vomiting and diarrhoea [11].

Outbreaks have been reported in a range of settings like restaurants and events with catered meals, nursing homes, hospitals, schools and vacation settings or cruise ships [12-15]. In Singapore, the first recorded outbreak of norovirus gastroenteritis was traced to the consumption of imported oysters [16]. As laboratory tests became more widely available, several other 
norovirus outbreaks in schools, nursing homes and army camps were reported [17-23].

In late December 2012 and early January 2013, the Ministry of Health (MOH), Singapore, was notified of two clusters of gastroenteritis in guests who attended two separate events at a hotel. Food served at the events had been catered by the hotel's Chinese banquet restaurant. Epidemiological investigations were immediately conducted to determine the aetiology of the outbreak and the modes of transmission. We report herein the findings of our investigations.

\section{METHODS AND MATERIALS}

\section{Epidemiological investigations}

A total of 15 separate events were held at the hotel between 23 and 31 December 2012. Interviews with the event organizers confirmed that attendees at six of the 15 events (including the two which had been reported to the $\mathrm{MOH}$ ) had developed acute gastroenteritis after attending the events. Guest lists were obtained from the organizers, after which all attendees were contacted and interviewed via telephone by trained public health officers. Epidemiological information elicited through a standardized questionnaire included personal particulars, clinical symptoms, onset of illness, food items consumed, medical treatment sought, and exposure to any person with diarrhoea or vomiting 3 days prior to illness onset.

\section{Case definition, control selection and statistical analysis}

A case was defined as a previously well individual who developed diarrhoea ( $\geqslant 2$ episodes within $24 \mathrm{~h}$ ) and/or vomiting with or without fever, nausea or abdominal pain after attending an event held at the hotel between 23 and 31 December 2012.

Six case-control studies were conducted in guests from the six affected events held at the hotel between 26 and 30 December 2012. Thirteen individuals with onset of illness $>72 \mathrm{~h}$ after attending the respective events were excluded from the statistical analyses as they exceeded the maximum known incubation period for norovirus [24]. All contactable asymptomatic guests were selected as controls. Univariate analysis was conducted to estimate the association between each food item and illness. Crude odds ratios (ORs) and their 95\% confidence intervals (CIs) were calculated for the association between illness and consumption of each food item served at the six events.
Statistical analyses were carried out using SPSS v. 22 (IBM Corp., USA). $P<0.05$ was considered statistically significant.

\section{Food and environmental investigations}

The $\mathrm{MOH}$ and the National Environment Agency inspected all six of the hotel's kitchens between 31 December 2012 and 3 January 2013. A total of 27 food samples/remnants (including 21 from the Chinese banquet kitchen) and 31 environmental samples were collected for microbial analyses. The environmental samples were taken from the kitchen (chopping boards, crockery, glassware, taps, hand wash basins) as well as from the staff and guest toilets (door knobs, taps, sinks, soap dispensers) near the ballrooms where the events were held.

\section{Microbial investigation}

The food and environmental samples collected from the hotel were tested for total aerobic plate count, faecal coliforms, norovirus and the standard bacteriological panel. All food handlers who worked at the hotel were referred to the Communicable Diseases Centre (CDC) for stool screening of foodborne pathogens.

We attempted to obtain stool samples from as many cases as possible up to 12 days after the respective events for testing of bacterial and viral foodborne pathogens. Sterile stool bottles with instructions on collection procedures were despatched to cases by a courier. Once available, stool samples were collected by the courier and despatched to the National Public Health Laboratory (NPHL) and the Singapore General Hospital microbiology laboratory for viral (norovirus and rotavirus) and bacterial (including Salmonella, Shigella, Campylobacter and Vibrio species) testing, respectively.

\section{Sample preparation, nucleic acid extraction, and detection of norovirus}

Stool samples were emulsified in $1 \mathrm{ml}$ sterile $1 \times$ phosphate-buffered saline and centrifuged for $10 \mathrm{~min}$ at $3000 \mathrm{rpm}$. Viral RNA was extracted directly from the clarified supernatant using QIAamp Viral RNA Mini kit (Qiagen, Germany) according to the manufacturer's instructions and eluted in $60 \mu$ elution buffer. Norovirus was detected by a multiplex real-time reverse transcription-polymerase chain reaction (RT-PCR) [25]. 


\section{Genotyping of norovirus}

Samples positive by multiplex real-time RT-PCR assay were selected for genotyping using a conventional RT-PCR assay, targeting the partial capsid ORF2 sequences [26]. Amplicons of appropriate size, 253 bp for GII noroviruses, were purified by using ExoSAP-IT (Affymetrix, USA) and sequenced at Axil Scientific Pte Ltd, Singapore. The genotype of each sample was determined by the web-based Norovirus Automated Genotyping Tool [27].

\section{Phylogenetic analysis}

Phylogenetic analysis of partial capsid sequences from this outbreak was performed alongside full-length capsid reference sequences representative of the GII.3, GII.4 and GII.6 lineages obtained from GenBank, as well as partial capsid sequences of norovirus community isolates obtained from routine surveillance performed at the NPHL. All sequences were aligned to each other in MAFFT, using the E-INS-i algorithm [28]. The evolutionary history was inferred by using the maximum-likelihood method based on the Tamura-Nei model [29]. The tree with the highest log likelihood (-9197.6163) was chosen. Initial tree(s) for the heuristic search were obtained automatically by applying Neighbour-Joining and BioNJ algorithms to a matrix of pairwise distances estimated using the maximum composite likelihood approach, and then selecting the topology with superior log likelihood value. A discrete Gamma distribution was used to model evolutionary rate differences between sites [five categories $(+\mathrm{G}$, parameter $=0.7749)]$. The rate variation model allowed for some sites to be evolutionarily invariable $([+\mathrm{I}], 37 \cdot 7610 \%$ sites). The analysis involved 50 nucleotide sequences. Codon positions included were 1 st + $2 \mathrm{nd}+3 \mathrm{rd}+$ non-coding. There were a total of 1647 positions in the final dataset. Evolutionary analyses were conducted in MEGA6 [30].

\section{RESULTS}

\section{Descriptive epidemiology}

Of 1590 guests who attended six events in the hotel between 26 and 30 December 2012, 453 fulfilled our case definition, giving an attack rate of $28.5 \%$ for symptomatic cases. Thirteen cases that developed symptoms $>72 \mathrm{~h}$ after attending the respective events had continued exposure to primary cases and represented a second wave of infection (secondary cases).
A summary of the six events is shown in Table 1 while the epidemic curve is shown in Figure 1. The cases ranged in age from 1 to 75 years (median 33 years, mean 37 years) with slightly more females affected (male-to-female ratio 1:1·2). The overall distribution by ethnicity was $93.4 \%$ Chinese, $4.4 \%$ Malay, $0 \cdot 2 \%$ Indian, and $2 \cdot 0 \%$ other ethnic groups.

The reported clinical symptoms were diarrhoea $(89 \cdot 4 \%)$, vomiting $(59 \cdot 4 \%)$, nausea $(56 \cdot 1 \%)$, fever $(53.0 \%)$ and abdominal pain (49.4\%). The incubation period based on the interval between time of food consumption and onset of illness ranged from $2 \mathrm{~h}$ to $70 \mathrm{~h}$ (median $38 \mathrm{~h}$, mean $37 \mathrm{~h}$ ) for primary cases, and ranged from $77 \mathrm{~h}$ to $162 \mathrm{~h}$ (median $103 \mathrm{~h}$, mean $109 \mathrm{~h}$ ) for secondary cases. Twenty-four primary cases reported incubation periods $<12 \mathrm{~h}$ (median $8 \mathrm{~h}$, mean $8 \mathrm{~h}$ ), which may have been due to recall bias as cases were interviewed more than 9 days after the events. Of the reported cases, $213(47 \cdot 0 \%)$ cases sought outpatient treatment, $190(42 \cdot 0 \%)$ self-medicated while $50(11.0 \%)$ recovered without any treatment. None of the cases were hospitalized.

\section{Analytical epidemiology}

Six case-control studies were conducted in guests from the six affected events. Case-control analyses showed a significant association between illness and consumption of 10 food items (Table 2). Consumption of prawn salad was identified to be significantly associated with illness in two of the four events at which it was served (events 2 and 6). Five of the remaining food items were significantly associated with only one specific event although they were served in two or more events while the rest were only served at one specific event each.

\section{Environmental and microbial findings}

Three cockroaches (including a nymph) were found during inspection of the Chinese banquet/restaurant kitchens.

A total of 46 stool samples were collected from 46 unique individuals across all the events except the fifth, from which stool samples could not be obtained. Of these, $34(73.9 \%)$ tested positive for norovirus GII. Seven of these 34 samples were genotyped to be norovirus strain GII.4 Sydney 2012 variant. The viral titre was too low for the rest of the samples to be genotyped. 


\begin{tabular}{|c|c|c|c|c|c|c|c|c|c|}
\hline \multirow{2}{*}{$\begin{array}{l}\text { Event } \\
\text { no. }\end{array}$} & \multirow[b]{2}{*}{ Date of event } & \multirow[b]{2}{*}{ Type of event } & \multirow{2}{*}{$\begin{array}{l}\text { No. of } \\
\text { cases* }^{*}\end{array}$} & \multirow{2}{*}{$\begin{array}{l}\text { No. of } \\
\text { attendees }\end{array}$} & \multirow{2}{*}{$\begin{array}{l}\text { Attack } \\
\text { rate }(\%) \dagger\end{array}$} & \multicolumn{2}{|c|}{$\begin{array}{l}\text { No. (\%) selected for } \\
\text { case-control study }\end{array}$} & \multirow[b]{2}{*}{ Type of food served } & \multirow[b]{2}{*}{ Source of food } \\
\hline & & & & & & Cases & Controls & & \\
\hline 1 & 26 Dec. 2012 & Wedding banquet & 109 & 290 & 37.6 & $89(81 \cdot 7)$ & $86(47 \cdot 5)$ & Pastries \& Chinese food & $\begin{array}{l}\text { Chinese banquet kitchen } \\
\& \text { pastry kitchen }\end{array}$ \\
\hline 2 & 27 Dec. 2012 & Wedding banquet & 108 & 330 & $32 \cdot 7$ & $106(98 \cdot 1)$ & $24(10 \cdot 8)$ & Pastries \& Chinese food & $\begin{array}{l}\text { Chinese banquet kitchen } \\
\& \text { pastry kitchen }\end{array}$ \\
\hline 3 & 28 Dec. 2012 & Corporate dinner & 69 & 220 & $31 \cdot 4$ & $65(94 \cdot 2)$ & $63(41 \cdot 7)$ & $\begin{array}{l}\text { Nuts, Chinese food, Chinese } \\
\text { vegetarian food \& } \\
\text { halal-certified food }\end{array}$ & $\begin{array}{l}\text { Chinese banquet kitchen } \\
\text { \& external vendor }\end{array}$ \\
\hline 4 & 29 Dec. 2012 & Wedding banquet & 63 & 330 & $19 \cdot 1$ & $61(96 \cdot 8)$ & $99(37 \cdot 1)$ & $\begin{array}{l}\text { Nuts, Chinese food \& Malay } \\
\text { food }\end{array}$ & Chinese banquet kitchen \\
\hline 5 & 29 Dec. 2012 & Wedding banquet & 10 & 100 & $10 \cdot 0$ & $10(100 \cdot 0)$ & $17(18 \cdot 9)$ & Nuts, Chinese food & Chinese banquet kitchen \\
\hline 6 & 30 Dec. 2012 & Wedding banquet & 94 & 320 & $29 \cdot 4$ & $90(95 \cdot 7)$ & $71(31 \cdot 4)$ & $\begin{array}{l}\text { Chinese food, Chinese } \\
\text { vegetarian food \& Malay } \\
\text { food }\end{array}$ & $\begin{array}{l}\text { Chinese banquet kitchen } \\
\text { \& Western kitchen }\end{array}$ \\
\hline
\end{tabular}

* Number of cases includes 13 secondary cases.

$\dagger$ The denominator for the attack rate includes individuals who did not fulfil the case definition based on the specified symptoms. 


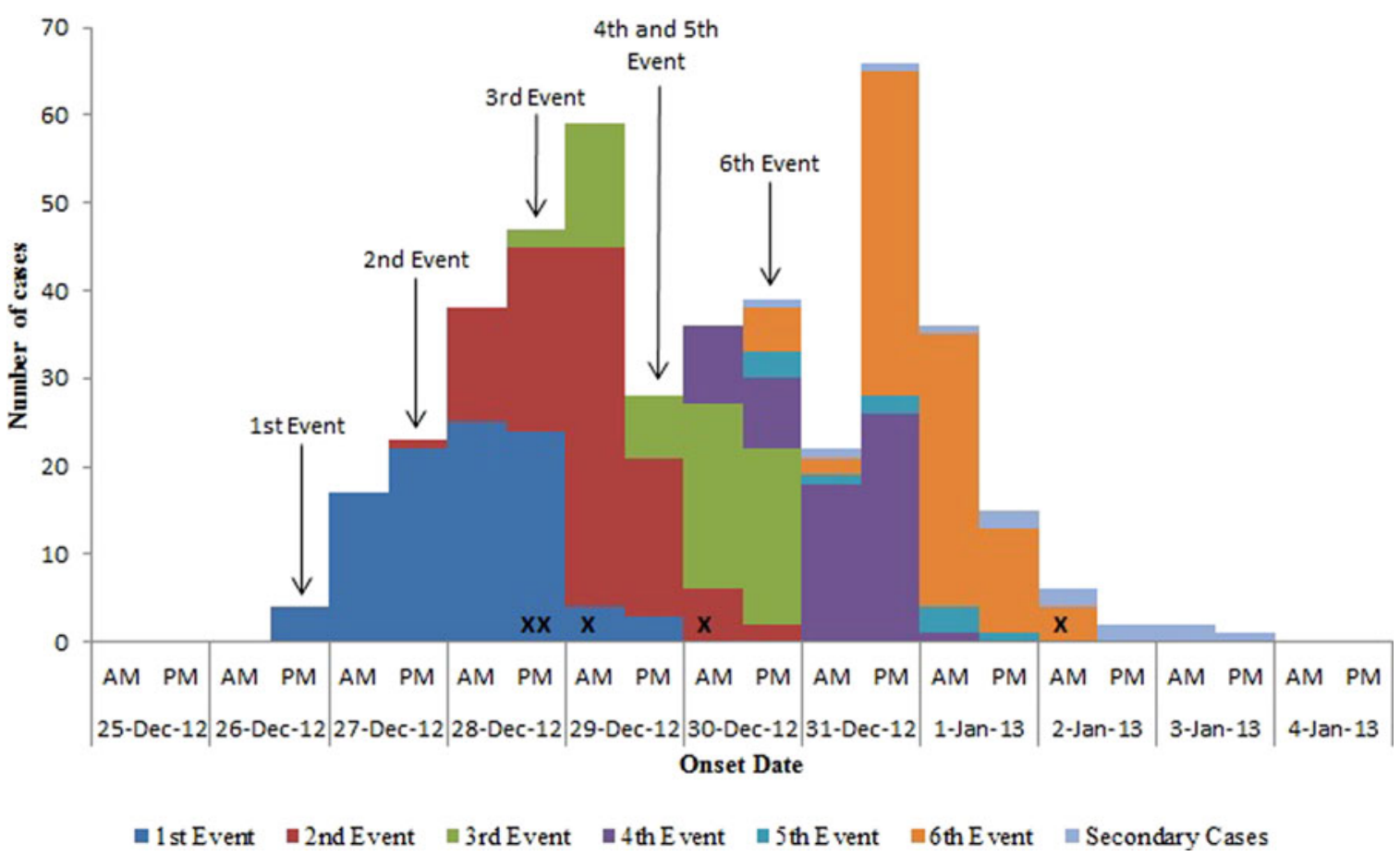

Fig. 1. Onset of illness of 441 reported cases (12 cases could not remember their date of onset of symptoms) in an outbreak of norovirus gastroenteritis, December 2012. X, Onset of illness of food handlers working at the Chinese banquet kitchen who tested positive for norovirus (excluding two asymptomatic food handlers). Each $\mathbf{X}$ represents one symptomatic food handler.

Table 2. Crude odds ratios for statistically significant association between illness and food items served at five of the six events in an outbreak of norovirus gastroenteritis in a hotel

\begin{tabular}{|c|c|c|c|c|c|c|c|c|}
\hline \multirow[b]{2}{*}{ Event/menu } & \multirow[b]{2}{*}{ Food items } & \multicolumn{2}{|c|}{ Cases } & \multicolumn{2}{|c|}{ Controls } & \multirow[b]{2}{*}{ Crude OR } & \multirow[b]{2}{*}{$95 \% \mathrm{CI}$} & \multirow[b]{2}{*}{$P$ value } \\
\hline & & Ate & $\%$ Ate & Ate & $\%$ Ate & & & \\
\hline \multirow{2}{*}{ 2/Chinese } & Prawn salad & 94 & $88 \cdot 7 \%$ & 16 & $66 \cdot 7 \%$ & 3.92 & $1 \cdot 39-11 \cdot 08$ & $0 \cdot 012$ \\
\hline & Chicken simmered in red wine & 94 & $88 \cdot 7 \%$ & 16 & $66 \cdot 7 \%$ & 3.92 & $1 \cdot 39-11 \cdot 08$ & $0 \cdot 012$ \\
\hline 3/Chinese & Spring roll & 44 & $86 \cdot 3 \%$ & 18 & $35 \cdot 3 \%$ & $11 \cdot 52$ & $4 \cdot 31-30 \cdot 79$ & $<0 \cdot 0005$ \\
\hline \multirow[t]{3}{*}{ 3/Halal } & Orange soda & 5 & $35 \cdot 7 \%$ & 0 & $0.0 \%$ & * & - & 0.042 \\
\hline & Tempura scallop with fried squid & 14 & $100 \cdot 0 \%$ & 7 & $58 \cdot 3 \%$ & * & - & $0 \cdot 012$ \\
\hline & Rice & 14 & $100 \cdot 0 \%$ & 7 & $58 \cdot 3 \%$ & * & - & 0.012 \\
\hline 4/Malay & Seafood fried rice & 6 & $100 \cdot 0 \%$ & 4 & $44 \cdot 4 \%$ & * & - & $0 \cdot 044$ \\
\hline \multirow[t]{3}{*}{ 6/Chinese } & Prawn salad & 87 & $98 \cdot 9 \%$ & 55 & $88 \cdot 7 \%$ & $11 \cdot 07$ & $1 \cdot 33-92 \cdot 46$ & $0 \cdot 009$ \\
\hline & Spicy jellyfish & 85 & $96 \cdot 6 \%$ & 40 & $64 \cdot 5 \%$ & $15 \cdot 58$ & $4 \cdot 41-55 \cdot 13$ & $<0.0005$ \\
\hline & Deep fried prawn & 85 & $96 \cdot 6 \%$ & 52 & $83 \cdot 9 \%$ & $5 \cdot 45$ & $1 \cdot 43-20 \cdot 72$ & $0 \cdot 008$ \\
\hline
\end{tabular}

OR, Odds ratio; CI, confidence interval.

* ORs could not be calculated due to zero cell values.

Eighty-three food handlers working in the hotel (76 from the hotel's kitchens and seven from an external vendor who operated the staff canteen and prepared food in-house) were referred to CDC for stool screening. Fifteen of them tested positive for norovirus GII, comprising seven working in the Chinese banquet kitchen, three from the Chinese restaurant kitchen, and five from the hotel main kitchen. One food handler from the Chinese banquet kitchen and another from the Chinese restaurant kitchen were genotyped to have norovirus strain GII.4 Sydney 2012 variant while the viral titre was too low for the rest of the samples to be genotyped.

Eight of the 15 food handlers had gastroenteritis symptoms between 18 December 2012 and 2 
January 2013, while the rest remained asymptomatic. The 15 norovirus-positive food handlers were involved in a range of duties such as handling raw food, preparation of hot and cold dishes, plating of food, cleaning of utensils, glassware and dishware, and preparation of beverages. Eight of the 15 food handlers, including four from the Chinese banquet kitchen, had continued to work although they were symptomatic. The putative index case was a food handler who had become ill on 18 December 2012, and had worked while symptomatic.

Although four of the norovirus-positive food handlers worked in two other kitchens which cater to the hotel's Chinese and international restaurants, neither the $\mathrm{MOH}$ nor the hotel were notified of any other outbreaks of gastroenteritis linked to the hotel's restaurants during this time period.

A total of 27 food samples/remnants and 31 environmental samples were collected between 31 December 2012 and 3 January 2013. Two food samples (soya sauce chicken and roasted pork) and two remnants (roasted chicken and cold dish platter) collected from the Chinese banquet kitchen were positive for faecal coliforms. Five food samples and three remnants had high aerobic plate counts and nine food samples and two remnants had high total coliforms.

Two food remnants (a cold dish platter and chilled rock melon cream with sago pearl and ice cream) served on 26 December 2012 and submitted by the hotel to a private laboratory for microbiological analyses were positive for Escherichia coli. Another two samples of the same food items served on 27 December 2012 also tested positive for E. coli.

None of the 31 environmental samples were positive for enterobacterial pathogens. Twenty-nine of the 31 swabs were tested for norovirus. All were negative.

Phylogenetic analysis of isolates from the outbreak, when compared with closely related GII.4 sequences as well as isolates obtained from community surveillance, showed a cluster of GII.4 strains from the Australasian region which the outbreak isolates clustered to, along with some community isolates obtained from the same time period (Fig. 2). Other community isolates clustered with other GII.4 lineages, and yet others clustered with GII.3 and GII.6, indicating the presence of different lineages circulating in the region. Of interest was that the GII.4 lineage to which the outbreak isolates clustered featured several reference strains from a very narrow window of about 2-3 years. While food handlers were indicated on the tree, the close identity of the sequenced capsid regions made it difficult to determine a chain of transmission, nor was it possible to phylogenetically distinguish community isolates from outbreak isolates.

\section{DISCUSSION}

The aetiology of this outbreak was confirmed by the detection of norovirus GII from $34(74 \%)$ of the 46 stool samples obtained from the cases. Fifteen of the 83 food handlers also tested positive for norovirus GII, of whom eight continued to work while symptomatic. Precise identification of a causative strain was not possible in this instance but phylogenetic analysis suggested a regionally circulating lineage which includes two variants of GII.4/Sydney/2012, which was first reported in March 2012 in Australia and has since caused acute gastroenteritis outbreaks in several countries [31].

The only common factor for all six events was that they had had food, crockery and cutlery provided or handled by the hotel's Chinese banquet kitchen. No common food item was identified to be significantly associated with illness across all six events. However, prawn salad was shown to be statistically significantly associated with illness in the second and sixth events, implying that the prawn salad could have been one of the vehicles of transmission of norovirus for the second and sixth events.

Seven norovirus-positive food handlers had worked at the Chinese banquet kitchen, including four who had continued working even while they had diarrhoea and/or vomiting. Two had remained asymptomatic and the remaining food handler had not worked while symptomatic. The food handlers who had worked while symptomatic may have been infectious with norovirus, which may have led to contamination of the food and environment. Consumption of contaminated food, especially food which has not been thoroughly cooked, such as salads, may then cause illness. Asymptomatic food handlers may also cause contamination of food, and have been reported as the sources of several norovirus outbreaks [32-36]. In this instance, the asymptomatic food handlers could have triggered and/or propagated the outbreak as we noted that apart from the putative index case, the other symptomatic food handlers working in the Chinese banquet kitchen only developed symptoms from the day of the third event onwards. The identification of E. coli, faecal coliforms and high bacterial counts in the food samples further supports the presence of poor hygiene and food handling practices. 


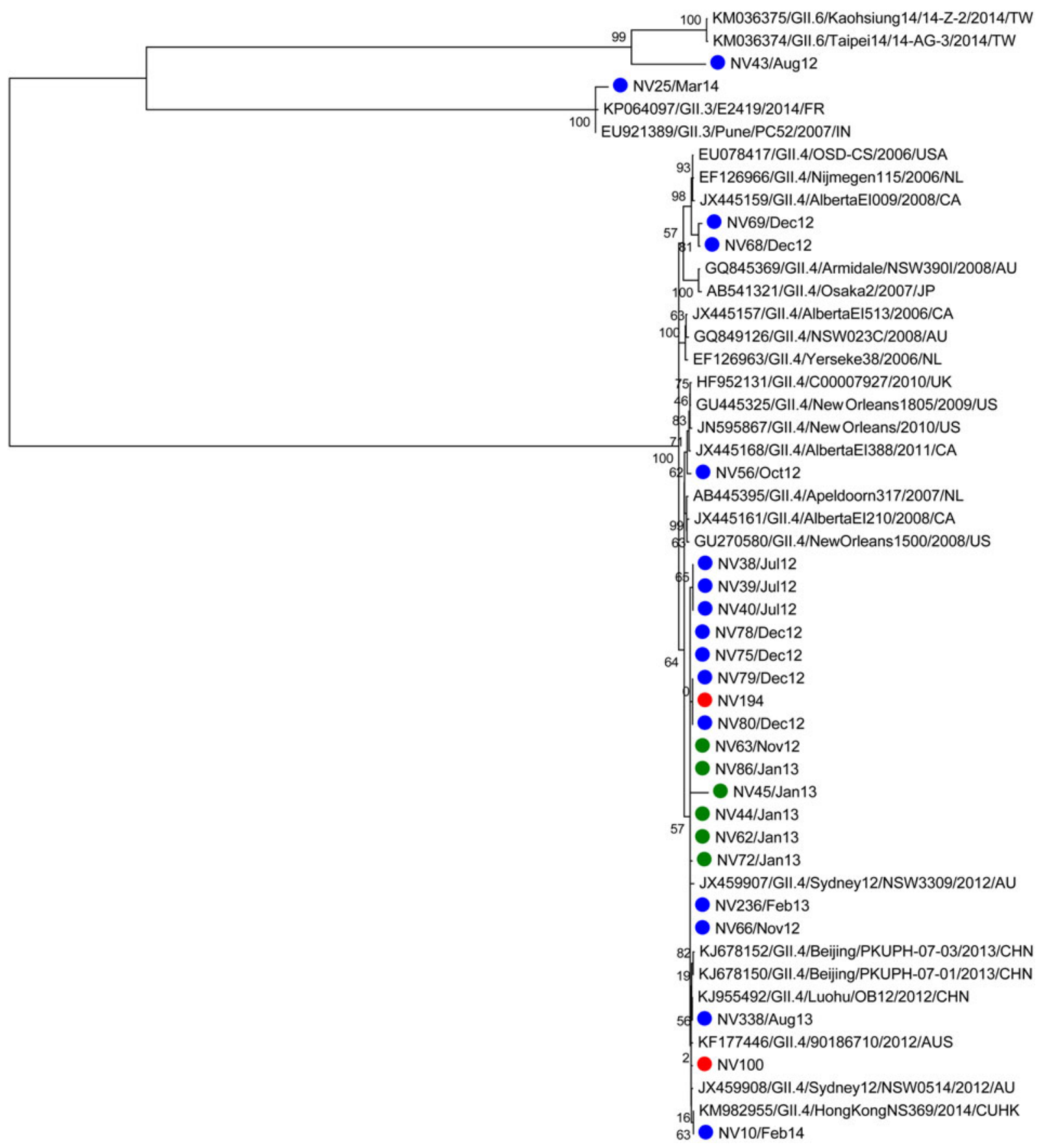

Fig. 2. Phylogenetic analysis of norovirus isolates from the outbreak. Representation of the phylogenetic relationship between outbreak isolates (green dots; food handlers indicated by red dots), community isolates (blue dots), and reference isolates (black text). The diagram shows the tree with the highest log likelihood out of 1000 bootstrapped iterations - the percentage of replicate trees in which the associated taxa clustered together in the bootstrap test is shown next to the branches.

However, it should be noted that not all the cases had consumed food catered by the Chinese banquet kitchen. Fifteen cases who had attended the third event had consumed halal-certified food which was catered from an external vendor. The vendor had provided separate plates and cutlery to be used by the guests consuming the halal-certified food. These items were wiped with cloths by food handlers from 
the Chinese banquet kitchen prior to usage. The drinking glasses that were served to all guests were from a common pool provided by the Chinese banquet kitchen and were also wiped with cloths before use. Three of the norovirus-positive food handlers, including two who were asymptomatic, had been involved in wiping the crockery and cutlery. All three had used their bare hands to hold the crockery and cutlery while wiping them with cloths. It has been shown that norovirus can be transferred between fingers and fomites [7, 37]. Hence, it is possible that these food handlers had contaminated the crockery and cutlery, and guests had become ill after using the contaminated cutlery or consuming food or drink which had come into contact with the contaminated crockery.

Three of the 15 norovirus-positive food handlers worked in neighbouring kitchens, and they could have become infected through person-to-person transmission, given that they worked in close quarters. They also used common staff toilets located near the kitchens, and could have also become infected through contact with contaminated environmental surfaces. The other five food handlers worked in a kitchen situated on a different floor, but also used the same staff toilets. None of the food handlers from the staff canteen tested positive for norovirus. Hence, it is unlikely that the food handlers from the hotel were infected through consumption of food from the staff canteen.

There are a number of limitations in the epidemiological investigations of this outbreak. As the organizers of the fifth event were overseas, we were only able to verify information pertaining to their event after they returned, 9 days after the event. We were also unable to organize the collection of stool samples from the affected individuals from that event for testing. Most guests of the events also had difficulty in recalling what they had eaten and the time of onset of their symptoms, due to the long interval between the event and interview (more than 9 days apart). In addition, as the organizer of the first event had contacted the hotel prior to notifying the authorities of the outbreak, we were unable to collect relevant food remnants for testing as the hotel had already disposed of the remnants after sending a few samples to their own laboratory for testing. Prawn salad, which was implicated in the second and sixth events of the case-control studies, was unavailable for testing. The cloths that were used to clean the plates, cutlery and glasses had also been discarded prior to field inspections conducted by the authorities.
The Chinese banquet and restaurant kitchens, which share the same premises and function adjacent to each other, were suspended from operating with effect from 3 January 2013, and were only allowed to resume operations on 6 February 2013 after they had rectified the identified food and environmental hygiene lapses, and had enhanced food-safety measures. Food handlers who had tested positive for foodborne pathogens were prohibited from working and were only allowed to resume work after they were confirmed to be asymptomatic and had two consecutive stool samples collected 1 week apart that tested negative for foodborne pathogens.

In conclusion, we have described a large commonsource outbreak of norovirus gastroenteritis involving attendees of six events at a hotel over a period of 5 days. The outbreak was most likely due to the consumption of food contaminated by infected food handlers, supported by the fact that the norovirus detected from reported cases and food handlers was of the same genogroup GII, and that the putative index case was a symptomatic food handler. While we could not implicate a specific food item that was common across all six events, and norovirus was not detected in any of the food items sampled, there was evidence of poor hygiene and food handling practices, as shown by the bacterial contamination of food. Notwithstanding the negative environmental samples, transmission through contact with contaminated environmental surfaces could also have occurred, as evidenced by the fact that several affected cases had not consumed food provided by the hotel's kitchen, but had used cutlery and crockery that had been handled by infected food handlers. This outbreak highlights the public health importance of ensuring that food handlers comply strictly with personal hygiene and food-safety practices, including refraining from working when they are ill with gastroenteritis symptoms, so as to minimize the risk of foodborne infections. Strict adherence to refraining from work while ill with gastroenteritis symptoms would have reduced the spread of norovirus infection in food handlers and could have prevented this outbreak from occurring.

\section{ACKNOWLEDGEMENTS}

The authors thank the staff of the Communicable Diseases Division, the National Public Health Laboratory and the Public Health Intelligence Branch from the Ministry of Health and the 
National Environment Agency for their help throughout the investigation.

\section{REFERENCES}

1. Koo HL, et al. Noroviruses: the principal cause of foodborne disease worldwide. Discovery Medicine 2010; 10: 61-70.

2. Centers for Disease Control and Prevention. Norovirus: worldwide (http://www.cdc.gov/norovirus/worldwide. html). Accessed 17 August 2015.

3. Ahmed SM, et al. Global prevalence of norovirus in cases of gastroenteritis: a systematic review and meta-analysis. Lancet Infectious Diseases 2014; 14: 725-730.

4. Koopmans M. Noroviruses in healthcare settings: a challenging problem. Journal of Hospital Infection 2009; 73: 331-337.

5. UpToDate. Epidemiology, clinical manifestations and diagnosis of norovirus and related viruses (http://www. uptodate.com/contents/epidemiology-clinical-manifestations-and-diagnosis-of-noroviruses-and-related-viruses). Accessed 17 August 2015.

6. Kimberly KR, William EK. A point-source norovirus outbreak caused by exposure to fomites. Journal of Infectious Diseases 2012; 205: 1639-1641.

7. Tuladhar E, et al. Transfer of noroviruses between fingers and fomites and food products. International Journal of Food Microbiology 2013; 167: 346-352.

8. Centers for Disease Control and Prevention. Norovirus: clinical overview (http://www.cdc.gov/norovirus/hcp/ clinical-overview.html). Accessed 17 August 2015.

9. Atmar RL, et al. Norwalk virus shedding after experimental human infection. Emerging Infectious Diseases 2008; 14: 1553-1557.

10. Glass RI, Parashar UD, Estes MK. Norovirus gastroenteritis. New England Journal of Medicine 2009; 361: 1776-1785.

11. Teunis PF, et al. Shedding of norovirus in symptomatic and asymptomatic infections. Epidemiology and Infection 2015; 143: 1710-1717.

12. Monroe SS, Ando T, Glass RI. Introduction: Human enteric caliciviruses-an emerging pathogen whose time has come. Journal of Infectious Diseases 2000; 181: 249-251.

13. Xerry $\mathbf{J}$, et al. Transmission events within outbreaks of gastroenteritis determined through analysis of nucleotide sequences of the P2. Journal of Clinical Microbiology 2008; 46: 947-953.

14. Kageyama T, et al. Coexistence of multiple genotypes, including newly identified genotypes, in outbreaks of gastroenteritis due to norovirus in Japan. Journal of Clinical Microbiology 2004; 42: 2988-2995.

15. European Centre for Disease Prevention and Control. Norovirus: factsheet for health professionals (http:// ecdc.europa.eu/en/healthtopics/norovirus_infection/ factsheet-health-professionals/Pages/factsheet_health_ professionals.aspx). Accessed 17 August 2015.
16. Ng TL, et al. Oyster-associated outbreaks of norovirus gastroenteritis in Singapore. Journal of Infection 2005; 51: 413-418.

17. Ho ZJ, et al. Emergence of norovirus GI.2 outbreaks in military camps in Singapore. International Journal of Infectious Diseases 2015; 31: 23-30.

18. Yap J, et al. Outbreak of acute norovirus gastroenteritis in a military facility in Singapore: A public health perspective. Singapore Medical Journal 2012; 53: 249-254.

19. Ler SS, et al. Norovirus outbreak at a primary school in Singapore. Epidemiological News Bulletin, Ministry of Health (Singapore) 2011; 37: 94-97.

20. Minn T, et al. Norovirus outbreak at a nursing home in Singapore. Epidemiological News Bulletin, Ministry of Health (Singapore) 2012; 38: 72-76.

21. Minn T, et al. Norovirus gastroenteritis outbreak at a nursing home in Singapore. Epidemiological News Bulletin, Ministry of Health (Singapore) 2013; 39: 40-44.

22. Tien WS, Hishamuddin P, Tay J. Outbreak of gastroenteritis in a junior college in Singapore. Epidemiological News Bulletin, Ministry of Health (Singapore) 2013; 39: 70-75.

23. Pang QY, et al. Outbreak of norovirus gastroenteritis at a nursing home in Singapore. Epidemiological News Bulletin, Ministry of Health (Singapore) 2013; 39: 95100.

24. Heymann DL (ed). Control of Communicable Diseases Manual. American Public Health Association, 2008, pp. 436-438.

25. Pang XL, Preiksaitis JK, Lee B. Multiplex real time RT-PCR for the detection and quantitation of norovirus genogroups I and II in patients with acute gastroenteritis. Journal of Clinical Virology 2005; 33: 168-171.

26. Vinjé J, Hamidjaja RA, Sobsey MD. Development and application of a capsid VP1 (region D) based reverse transcription PCR assay for genotyping of genogroup I and II noroviruses. Journal of Virological Methods 2004; 116: 109-117.

27. Kroneman A, et al. An automated genotyping tool for enteroviruses and noroviruses. Journal of Clinical Virology 2011; 51: 121-125.

28. Katoh K, Standley DM. MAFFT multiple sequence alignment software version 7: improvements in performance and usability. Molecular Biology and Evolution 2013; 30: 772-780.

29. Tamura K, Nei M. Estimation of the number of nucleotide substitutions in the control region of mitochondrial DNA in humans and chimpanzees. Molecular Biology and Evolution 1993; 10: 512-526.

30. Tamura K, et al. MEGA6: Molecular Evolutionary Genetics Analysis version 6.0. Molecular Biology and Evolution 2013; 30: 2725-2729.

31. Van Beek $\mathbf{J}$, et al. Indications for worldwide increased norovirus activity associated with emergence of a new variant of genotype II.4, late 2012. Eurosurveillance 2013; 18: 8-9.

32. Kuo HW, et al. A non-foodborne norovirus outbreak among school children during a skiing holiday, Austria, 2007. Wiener Klinische Wochenschrift 2009; 121: $120-124$. 
33. Barrabeig I, et al. Foodborne norovirus outbreak: the role of an asymptomatic food handler. $B M C$ Infectious Diseases 2010; 10: 269.

34. Schmid D, et al. Foodborne gastroenteritis outbreak in an Austrian healthcare facility caused by asymptomatic, norovirus-excreting kitchen staff. Journal of Hospital Infection 2011; 77: 237-241.

35. Nicolay $\mathbf{N}$, et al. Potential role of asymptomatic kitchen food handlers during a food-borne outbreak of norovirus infection, Dublin, Ireland, March 2009. Eurosurveillance 2011; 16: pii $=19931$.

36. Jeong AY, et al. Occurrence of norovirus infections in asymptomatic food handlers in South Korea. Journal of Clinical Microbiology 2013; 51: 598-600.

37. Liu P, et al. Laboratory evidence of Norwalk virus contamination on the hands of infected individuals. Applied and Environmental Microbiology 2013; 79: 7975-7981. 